\title{
Adenoma detection rate in 41,010 patients from Southwest China
}

\author{
BIN CAI ${ }^{1}$, ZHIXIAN LIU $^{1}$, YANSONG XU $^{2}$, WEIYUAN WEI ${ }^{3}$ and SEN ZHANG $^{1}$ \\ Departments of ${ }^{1}$ Colorectal Surgery; ${ }^{2}$ Emergency Surgery and ${ }^{3}$ Gastrointestinal Surgery, \\ The First Affiliated Hospital of Guangxi Medical University, Nanning, Guangxi 530021, P.R. China
}

Received May 28, 2014; Accepted February 13, 2015

DOI: $10.3892 / \mathrm{ol} .2015 .3005$

\begin{abstract}
Adenoma detection rate (ADR) is considered as an important predictor of the risk of interval colorectal cancer following a screening colonoscopy. A retrospective review of all the patients who underwent colonoscopy in the First Affiliated Hospital of Guangxi Medical University (Nanning, China) between 2003 and 2012 was performed, with the aim of estimating the ADR in Southwest China and determine the effects of age, gender and polyp location on ADR. Demographic information and data regarding the timing of the colonoscopy, number of polyps removed, polyp location and pathological types of polyps were collected and analyzed. The patients were grouped according to colonoscopy date, polyp location, gender and age. The $\chi^{2}$ test was used to compare the ADR between the different age and gender groups and the Cochran-Armitage trend test was used to calculate the statistical significance of the ADR trend across age groups. A two-tailed value of $\mathrm{P}<0.05$ was considered to indicate statistically significant differences. A total of 41,010 patients were included, of whom 7,219 were diagnosed with at least one adenoma on pathological examination. Therefore, the ADR value of the 41,010 patients screened in the present study was $17.6 \%$. There was a statistically significant trend of increasing ADR with increasing age in both genders $(\mathrm{P}<0.05)$. In addition, the ADR of male patients was significantly higher compared with that of female patients in all age groups (all $\mathrm{P}<0.05)$. The ADR of patients aged $>50$ years was significantly higher compared with that of patients aged $<50$ years (28.8 vs. $11.0 \%$, respectively; $\mathrm{P}<0.05)$. In addition, $20 \%$ of the cases of detected adenomas occurred in the rectum, $47 \%$ in the left colon and $33 \%$ in the right colon.
\end{abstract}

Correspondence to: Dr Sen Zhang, Department of Colorectal Surgery, The First Affiliated Hospital of Guangxi Medical University, 22 Shuangyong Road, Nanning, Guangxi 530021, P.R. China

E-mail: zs1770@126.com

Key words: colorectal polyp, adenoma, adenoma detection rate, endoscopy, oncology

\section{Introduction}

A colorectal polyp is a circumscribed mass of tissue that projects above the surface of the bowel mucosa (1). Colorectal polyps are histologically classified as neoplastic (adenomas) or non-neoplastic (2). Adenomatous polyps exhibit a malignant potential and are classified as tubular, tubulovillous or villous adenomas, depending on the presence and volume of villous tissue. Adenoma detection rate (ADR) is defined as the number of patients with adenomas identified per 100 patients screened and is considered as an important predictor of the risk of interval colorectal cancer (CRC) following a screening colonoscopy (3). Several studies on ADR have been previously conducted. A nationwide survey of 3,266 patients in France reported an ADR of $17.7 \%(578 / 3,266)$, whereas the ADR of male patients was significantly higher compared with that of female patients ( 21.2 vs. $14.5 \%$, respectively; $\mathrm{P}<0.0001)$; furthermore, the ADR of patients aged $>50$ years was significantly higher compared with that of patients aged $<50$ years (20.6 vs. 8.5\%, respectively; $\mathrm{P}<0.0001$ ) (4). Another French study reported an ADR of $31 \%$ (5). In addition, a nationwide free colonoscopy screening program was conducted in Germany for the general population aged $\geq 55$ years. That study included 2,821,392 screening colonoscopies performed between January, 2003 and December, 2008 and reported an ADR of $19.4 \%$, with a higher rate in men compared with that in women (25.8 vs. $16.7 \%$, respectively) (6).

$\mathrm{CRC}$ is a high-morbidity malignant tumor with a high incidence worldwide, accounting for $8 \%$ of new cancer cases in the USA in 2014 (7). The majority of CRCs arise from pre-existing adenomatous polyps. The causal association between polypoid adenomas and colonic carcinomas has been demonstrated on a pathological basis $(8,9)$. Currently, the key to controlling $\mathrm{CRC}$ is reliably detecting and resecting adenomas prior to them becoming malignant. Colonoscopy is the most common and efficient method for the detection and removal of colorectal adenomas. ADR is considered to be an important factor affecting the risk of CRC. A higher ADR may reduce the incidence of $\mathrm{CRC}$, thereby protecting asymptomatic average-risk individuals against $\mathrm{CRC}$. A retrospective review of all the patients who underwent colonoscopy screening between 2003 and 2012 in the First Affiliated Hospital of Guangxi Medical University (Nanning, China) was conducted, with the aim of estimating the total ADR, comparing the ADR among the different gender and age groups and calculating the proportion of adenomatous polyps in each section of the large bowel. 


\section{Patients and methods}

Patients and data collection. All the patients who underwent colonoscopy at the First Affiliated Hospital of Guangxi Medical University between January, 2003 and December, 2012 were recruited in the present study. All the procedures were performed by endoscopists experienced in operational colonoscopy. All the patients underwent sufficient bowel preparations and all the removed specimens were pathologically examined. Data collected from the colonoscopy and pathology reports included demographic information, timing of the colonoscopy, number of polyps removed, polyp location and the pathological types of the polyps. The patients were grouped according to the colonoscopy date (2003-2012), gender (male and female), age ( $<30,30-39,40-49,50-59,60-69,70-79$ and $\geq 80$ years) and polyp location (ileocecum, ascending, transverse, descending and sigmoid colon and rectum).

The exclusion criteria were as follows: A history of colonoscopy and diagnosis of colorectal pathology; a history of CRC; a history of colorectal surgery; suboptimal bowel preparation; pathology report not available; and non-Chinese patients. The results were collected and reviewed. Patients with multiple adenomas were recorded only once when calculating the ADR. However, when calculating the proportion of adenomatous polyps in each location, for multiple polyps occurring in more than one location, each location was recorded once. For example, if adenomatous polyps were identified in the sigmoid colon and rectum of a patient, this patient was added to the sigmoid colon as well as the rectum groups.

Statistical analysis. The patient characteristics are described as frequencies and percentages for the categorical variables. The $\chi^{2}$ test was used to compare the ADR between different age and gender groups. The statistical significance of the trend of ADR across different age groups was calculated using the Cochran-Armitage trend test. A two-tailed P-value of $<0.05$ was considered to indicate statistically significant differences. All the analyses were performed using SPSS 17.0 software (SPSS Inc., Chicago, IL, USA).

\section{Results}

$A D R$. Between 2003 and 2012, a total of 41,010 patients (20,210 men and 20,800 women) underwent electronic colonoscopy in our hospital and 7,219 (4,504 men and 2,715 women) were diagnosed with at least one adenoma on pathological examination. Therefore, the ADR of the patients screened in the present study was $17.6 \%$. The patient demographics per year are summarized in Table I. The ADR ranged between 12.7 and $20.1 \%$ within these 10 years, whereas no significant difference in ADR was observed after 10 years. The ADR in male and female patients per age group is presented in Table II. The ADR increased from $7.8 \%$ in patients aged $<30$ years to $48.4 \%$ in those aged $>80$ years. There was a statistically significant trend of increasing ADR with each decade of age in both genders $(\mathrm{P}<0.05)$ (Fig. 1). Furthermore, the ADR of patients aged $>50$ years was significantly higher compared with that of patients aged $<50$ years ( 28.8 vs. $11.0 \%$, respectively; $\mathrm{P}<0.05$ ). In addition, in all the age groups, the ADR of male patients was significantly higher compared with that of female patients

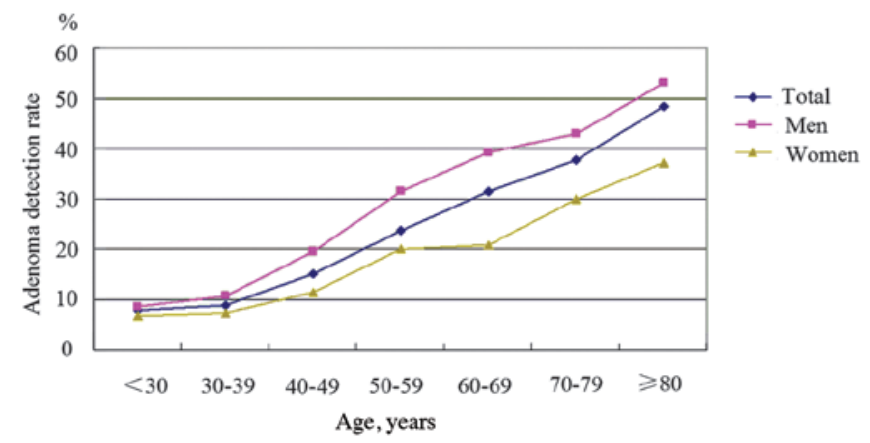

Figure 1. Trend of adenoma detection rate with the increase by decade of age. There was a statistically significant trend of increasing adenoma detection rate with increasing age in both genders $(\mathrm{P}<0.05)$.

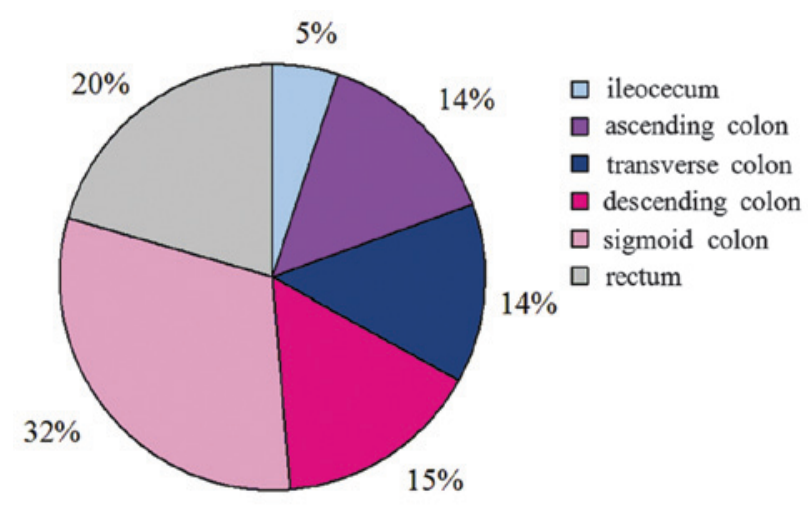

Figure 2. Proportion of adenomatous polyps in each section of the bowel. The left side of the colon includes the descending and sigmoid colon and the right side includes the ileocecum and the ascending and transverse colon.

(all $\mathrm{P}<0.05$ ), with a total ADR of $22.3 \%$ in men and $13.1 \%$ in women. For patients aged $>50$ years, the ADR was $37.0 \%$ in men and $20.6 \%$ in women.

Adenomas. Of the 7,219 patients diagnosed with at least one adenoma, 830 had adenomas in more than one location. In accordance with the aforementioned statistical method, there were a total of 8,551 cases of adenomas in the present study. The statistical analysis revealed that 433 cases of adenomas were detected in the ileocecum (5.1\%), 1,236 in the ascending colon (14.5\%), 1,155 in the transverse colon (13.5\%), 1,321 in the descending colon (15.4\%), 2,654 in the sigmoid colon $(31.0 \%)$ and 1,752 in the rectum (20.5\%). The proportion of adenomatous polyps in each section is depicted in Fig. 2, with $20 \%$ of the cases of adenomas occurring in the rectum, $47 \%$ in the left colon (descending and sigmoid colon) and $33 \%$ in the right colon (ileocecum, ascending and transverse colon).

\section{Discussion}

CRC is the third most common cause of cancer-related mortality in the USA (7). From a histological and endoscopic standpoint, CRC begins as a small neoplastic polyp, which progressively enlarges and transforms through a dysplastic stage into invasive cancer (10). The multistep progression from adenoma to cancer requires almost a decade or more and is 
Table I. Patient demographics per year.

\begin{tabular}{|c|c|c|c|c|c|}
\hline \multirow[b]{2}{*}{ Year } & \multicolumn{2}{|c|}{ Screened cases, no. } & \multicolumn{2}{|c|}{ Adenoma cases, no. } & \multirow[b]{2}{*}{$\operatorname{ADR}(\%)$} \\
\hline & Men & Women & Men & Women & \\
\hline 2003 & 989 & 1,075 & 170 & 93 & 12.7 \\
\hline 2004 & 1,135 & 1,280 & 215 & 157 & 15.4 \\
\hline 2005 & 1,422 & 1,505 & 303 & 189 & 16.8 \\
\hline 2006 & 1,967 & 2,112 & 437 & 256 & 17.0 \\
\hline 2007 & 1,876 & 1,926 & 498 & 266 & 20.1 \\
\hline 2008 & 2,267 & 2,389 & 474 & 276 & 16.1 \\
\hline 2009 & 2,321 & 2,304 & 506 & 329 & 18.1 \\
\hline 2010 & 2,607 & 2,489 & 604 & 343 & 18.6 \\
\hline 2011 & 2,647 & 2,751 & 679 & 382 & 19.7 \\
\hline 2012 & 2,979 & 2,969 & 618 & 424 & 17.5 \\
\hline
\end{tabular}

ADR, adenoma detection rate.

Table II. ADR for male and female patients in each age group.

\begin{tabular}{|c|c|c|c|c|c|c|c|}
\hline \multirow{2}{*}{$\begin{array}{l}\text { Age grouping } \\
\text { (years) }\end{array}$} & \multicolumn{3}{|c|}{ Screened cases, no. } & \multicolumn{3}{|c|}{ Adenoma cases, no. (ADR, \%) } & \multirow[b]{2}{*}{ P-value } \\
\hline & Total & Men & Women & Total & Men & Women & \\
\hline \multicolumn{8}{|l|}{ By decade } \\
\hline$<30$ & 6,338 & 3,428 & 2,910 & $496(7.8)$ & $299(8.7)$ & $197(6.8)$ & $<0.001$ \\
\hline $30-39$ & 9,402 & 4,465 & 4,937 & $834(8.9)$ & $473(10.6)$ & $361(7.3)$ & $<0.001$ \\
\hline $40-49$ & 10,116 & 4,654 & 5,462 & $1,525(15.1)$ & $910(19.6)$ & $615(11.3)$ & $<0.001$ \\
\hline $50-59$ & 7,785 & 3,566 & 4,219 & $1,834(23.6)$ & $1,122(31.5)$ & $712(20.0)$ & $<0.001$ \\
\hline $60-69$ & 4,628 & 2,414 & 2,214 & $1,451(31.4)$ & $946(39.2)$ & $505(20.9)$ & $<0.001$ \\
\hline $70-79$ & 2,293 & 1,364 & 929 & $862(37.6)$ & $585(42.9)$ & $277(29.8)$ & $<0.001$ \\
\hline$\geq 80$ & 448 & 319 & 129 & $217(48.4)$ & $169(53.0)$ & $48(37.2)$ & 0.003 \\
\hline \multicolumn{8}{|l|}{$\begin{array}{l}\text { By the cut-off } \\
\text { of } 50 \text { years }\end{array}$} \\
\hline$<50$ & 25,856 & 12,547 & 13,309 & $2,855(11.0)$ & $1,682(13.4)$ & $1,173(9.3)$ & $<0.001$ \\
\hline$\geq 50$ & 15,154 & 7,663 & 7,491 & $4,364(28.8)$ & $2,822(37.0)$ & $1,542(20.6)$ & $<0.001$ \\
\hline Total & 41,010 & 20,210 & 20,800 & $7,219(17.6)$ & $4,504(22.3)$ & $2,715(13.1)$ & $<0.001$ \\
\hline
\end{tabular}

ADR, adenoma detection rate.

accompanied by a number of characterized genetic alterations, involving mutation of the adenomatous polyposis coli (APC), deleted in colorectal carcinoma (DCC), Kirsten rat sarcoma viral oncogene homolog (K-Ras) and p53 genes, loss of heterozygosity (LOH) and DNA methylation.

APC gene mutation plays a significant role in the development of colorectal adenoma and CRC (11). APC is a tumor suppressor gene located on chromosome 5q. Germline mutation of the APC gene and subsequent somatic mutation of the second APC allele are responsible for the inherited familial adenomatous polyposis syndrome (12). There is evidence that the majority of CRCs and adenomas contain a mutated APC gene and mutations of the APC gene play a major role in the early development of colorectal neoplasms (13). It has been suggested that the APC protein may serve as a molecular 'gatekeeper' for the development of adenomas (14). A mutation of the 'gatekeeper' leads to a permanent imbalance of cell division over cell death and disrupts the cell-cell and cell-matrix interactions, leading to inappropriate cell proliferation.

$\mathrm{K}$-Ras gene activation is also considered to be an early event in colorectal carcinogenesis. It was previously reported that K-Ras mutations were detected in $58 \%$ of aberrant crypt foci (ACF) (15). Furthermore, ACF occured with a high frequency in the colons of animals treated with colon carcinogens and in the grossly normal mucosas of patients with colon cancer (16). In addition, the abnormal proteins produced by the K-Ras oncogene were frequently detected in colorectal neoplasms. These abnormal proteins may continually stimulate downstream 
signal transduction pathways involved in cell proliferation and malignant transformation.

$\mathrm{LOH}$ on the long arm of chromosome 18 (18q) is frequently observed in CRCs and advanced adenomas, but only occasionally in earlier-stage adenomas (17), indicating that $\mathrm{LOH}$ on $18 \mathrm{q}$ occurs later during the development from adenoma to carcinoma and may predict a poor prognosis. LOH on $18 \mathrm{q}$ was found to be closely associated with the inactivation of the DCC tumor suppressor gene. The DCC gene is located on chromosome 18q21.2 and contained within the common region $\mathrm{LOH}$ on 18q. DCC expression was found to be markedly decreased or absent in the majority of CRCs and cell lines. The DCC gene is one of the most commonly mutated tumor suppressor genes in CRC and occurs mainly in the late phase of carcinogenesis (18). The DCC protein is a transmembrane glycoprotein similar to the neural cell adhesion molecules (19). LOH on DCC may result in impaired contact between cells, thereby contributing to tumor growth and invasion (20). More recent evidence suggests that other tumor suppressor genes, namely deleted in pancreatic carcinoma 4 and Mad-related 2, may also be inactivated by allelic loss on chromosome $18 \mathrm{q}$ (21). In addition, mutation of the p53 tumor suppressor gene on chromosome $17 \mathrm{p}$ has been identified in approximately half of all CRCs and also frequently emerges in the later stages of colorectal carcinogenesis (22). This mutation may allow a growing tumor with multiple genetic alterations to evade cell cycle arrest and apoptosis.

In the present retrospective study, the ADR of the total 41,010 patients was $17.6 \%$. The ADR in patients aged $>50$ years was $28.8 \%(4,364 / 15,154)$, which was significantly higher compared with that in patients aged $<50$ years $(11.0 \%)$. These results are in accordance with most national guidelines, which recommend that screening programs for CRC be initiated by the age of 50 years for men and women of average risk, as the risk of CRC increases in the sixth decade of life (23). In addition, similar to the results of previous studies, the total ADR in men was found to be significantly higher compared with that in women ( 22.3 vs. $13.1 \%$, respectively) in the present study. In patients aged $>50$ years, the ADR was $37.0 \%$ in men and $20.6 \%$ in women. Based on the differences in ADR according to gender, it has been suggested that the age for initiating screening colonoscopy should be gender-specific. In the present study, the ADR of male patients was $19.6 \%$ in the 40-49 age group, whereas the ADR of female patients was $20.0 \%$ in the $50-59$ age group and $20.9 \%$ in the $60-69$ age group. This result was consistent with a previous study, in which comparable ADRs were found in men aged 45-49 and women aged 55-59 years (24). Sung et al (25) suggested in the Asia Pacific consensus recommendations for CRC screening that female subjects may start screening at a later age, due to the relatively low incidence of CRC at ages 50-55 years. It was previously reported that the higher incidence in men may be due to the longer female life span (26). Other studies suggested that the length of exposure to estrogen and/or progesterone may play a role in the gender difference $(27,28)$. In addition, other factors may also affect adenoma prevalence, including family history of colon cancer, tobacco use, obesity and diabetes mellitus (29). In clinical practice, the ADR was also found to be closely associated with the quality of bowel preparation, the cecal intubation rate, the level of operating techniques of the endoscopists and the quality of the endoscopic devices (4).
These factors may lead to missed adenomatous polyps or incompletely resected adenomas.

In the present study, $47 \%$ of the detected adenomas occurred in the left and $33 \%$ in the right colon. This result was in agreement with a previous study, in which left-sided colorectal adenomas were more prevalent compared with right-sided ones (56 vs. 44\%, respectively) (30). Another previous study, including a large sample of 7,590 adenomatous polyps, found that $76.5 \%$ of the adenomas were localized in the left and $23.5 \%$ in the right colon (31). The precise reason for this phenomenon is not fully understood. Fundamentally, the colon develops from two different embryonic areas of the primitive gut, namely the midgut, which gives rise to the small intestine through to the proximal two-thirds of the transverse colon, and the hindgut, which gives rise to the distal third of the transverse colon through to the upper anal canal (32). A previous study demonstrated that polyps with advanced pathology are smaller and more easily missed in the right compared with the left colon (33). In addition, the difficulty in detecting right-sided polyps, including suboptimal bowel preparation, flat polyps located behind mucosal folds and the shape of the colonic folds, may lead to missed adenomas. Recent studies have demonstrated that colonoscopy is significantly less effective in preventing proximal colon cancer and related mortality compared with distal colon cancer $(3,5)$.

The present study had certain limitations that must be acknowledged: i) The study was completed in a single academic center, which may make the present results only representative of a regional situation; ii) information regarding the size and type of adenomas were not available; therefore, they could not be further subdivided; iii) all the patients included were Chinese; therefore, the present results had racial limitations; iv) although all the colonoscopy examinations were conducted by experienced endoscopists, certain adenomas were inevitably missed; and v) traditionally, the left colon includes the ileocecum, ascending colon and proximal transverse colon and the right colon includes the distal transverse, descending and sigmoid colon. However, in the present retrospective study, the exact locations of the adenomas in the transverse colon could not be verified; therefore, all the adenomas in the transverse colon were classified into the left colon group. This means that the distribution ratio of adenomas in the right colon may in fact be higher than what was calculated in this study.

Despite the abovementioned limitations, the present study also has several strengths: All the data included in this study were rigorously reviewed. The study included a large sample of 41,010 patients, so that each group contained a sufficient number of samples for comparison.

In conclusion, in this retrospective study of 41,010 Chinese patients, an ADR of $17.6 \%$ was calculated. Adenomas were found to be more prevalent in male compared with female patients. In addition, regardless of gender, there was a statistically significant trend of increasing ADR with increasing age in this study.

\section{References}

1. Bond JH: Polyp guideline: diagnosis, treatment and surveillance for patients with nonfamilial colorectal polyps. The Practice Parameters Committee of the American College of Gastroenterology. Ann Intern Med 119: 836-843, 1993. 
2. Fenoglio-Preiser CM and Hutter RV: Colorectal polyps: pathologic diagnosis and clinical significance. CA Cancer J Clin 35: 322-344, 1985.

3. Kaminski MF, Regula J, Kraszewska E, et al: Quality indicators for colonoscopy and the risk of interval cancer. N Engl J Med 362 1795-1803, 2010.

4. Barret M, Boustiere C, Canard JM, et al; Société Française d'Endoscopie Digestive: Factors associated with adenoma detection rate and diagnosis of polyps and colorectal cancer during colonoscopy in France: results of a prospective, nationwide survey. PloS One 8: e68947, 2013.

5. Coriat R, Lecler A, Lamarque D, et al: Quality indicators for colonoscopy procedures: a prospective multicentre method for endoscopy units. PloS One 7: e33957, 2012.

6. Pox CP, Altenhofen L, Brenner H, Theilmeier A, Von Stillfried D and Schmiegel W: Efficacy of a nationwide screening colonoscopy program for colorectal cancer. Gastroenterology 142: 1460-1467, 2012.

7. Siegel R, Ma J, Zou Z and Jemal A: Cancer statistics, 2014. CA Cancer J Clin 64: 9-29, 2014.

8. Morson B: President's address. The polyp-cancer sequence in the large bowel. Proc R Soc Med 67: 451-457, 1974.

9. Hill MJ, Morson BC and Bussey HJ: Aetiology of adenoma-carcinoma sequence in large bowel. Lancet 1: 245-247, 1978.

10. Allen JI: Molecular biology of colon polyps and colon cancer. Semin Surg Oncol 11: 399-405, 1995.

11. Nakamura Y: The adenomatous polyposis coli gene and human cancers. J Cancer Res Clin Oncol 121: 529-534, 1995.

12. Gryfe R, Swallow C, Bapat B, Redston M, Gallinger S and Couture J: Molecular biology of colorectal cancer. Curr Probl Cancer 21: 233-300, 1997.

13. Powell SM, Zilz N, Beazer-Barclay Y, et al: APC mutations occur early during colorectal tumorigenesis. Nature 359: 235-237, 1992.

14. Kinzler KW and Vogelstein B: Lessons from hereditary colorecta cancer. Cell 87: 159-170, 1996.

15. Yamashita N, Minamoto T, Ochiai A, Onda M and Esumi $\mathrm{H}$ : Frequent and characteristic K-ras activation and absence of p53 protein accumulation in aberrant crypt foci of the colon. Gastroenterology 108: 434-440, 1995.

16. Pretlow TP, Brasitus TA, Fulton NC, Cheyer C and Kaplan EL: K-ras mutations in putative preneoplastic lesions in human colon. J Nat Cancer Inst 85: 2004-2007, 1993.

17. Vogelstein B, Fearon ER, Hamilton SR, et al: Genetic alterations during colorectal-tumor development. N Engl J Med 319: 525-532, 1988.

18. Fearon ER and Vogelstein B: A genetic model for colorectal tumorigenesis. Cell 61: 759-767, 1990.

19. Cho KR and Fearon ER: DCC: linking tumour suppressor genes and altered cell surface interactions in cancer? Eur J Cancer 31 A 1055-1060, 1995.

20. Chang SC, Lin JK, Lin TC and Liang WY: Loss of heterozygosity: an independent prognostic factor of colorectal cancer. World J Gastroenterol 11: 778-784, 2005.
21. Wang W, Li YF, Sun XW, et al: Correlation analysis between loss of heterozygosity at chromosome $18 \mathrm{q}$ and prognosis in the stage-II colon cancer patients. Chin J Cancer 29: 761-767, 2010

22. Baker SJ, Fearon ER, Nigro JM, et al: Chromosome 17 deletions and p53 gene mutations in colorectal carcinomas. Science 244: 217-221, 1989.

23. Levin B, Lieberman DA, McFarland B, et al; American College of Radiology Colon Cancer Committee: Screening and surveillance for the early detection of colorectal cancer and adenomatous polyps, 2008: a joint guideline from the American Cancer Society, the US Multi-Society Task Force on Colorectal Cancer and the American College of Radiology. Gastroenterology 134: 1570-1595, 2008.

24. Ferlitsch M, Reinhart K, Pramhas S, et al: Sex-specific prevalence of adenomas, advanced adenomas and colorectal cancer in individuals undergoing screening colonoscopy. JAMA 306: 1352-1358, 2011

25. Sung JJ, Lau JY, Young GP, et al; Asia Pacific Working Group on Colorectal Cancer: Asia Pacific consensus recommendations for colorectal cancer screening. Gut 57: 1166-1176, 2008.

26. Regula J, Rupinski M, Kraszewska E, et al: Colonoscopy in colorectal-cancer screening for detection of advanced neoplasia. N Engl J Med 355: 1863-1872, 2006.

27. Chlebowski RT, Wactawski-Wende J, Ritenbaugh C, et al; Women's Health Initiative Investigators: Estrogen plus progestin and colorectal cancer in postmenopausal women. $\mathrm{N}$ Engl J Med 350: 991-1004, 2004

28. Wei EK, Colditz GA, Giovannucci EL, Fuchs CS and Rosner BA: Cumulative risk of colon cancer up to age 70 years by risk factor status using data from the Nurses' Health Study. Am J Epidemiol 170: 863-872, 2009.

29. Dominic OG, McGarrity T, Dignan M and Lengerich EJ: American College of Gastroenterology Guidelines for Colorectal Cancer Screening 2008. Am J Gastroenterol 104 2626-2627; author reply 2628-2629, 2009.

30. Khodadoostan M, Fatemi R, Maserat E, et al: Clinical and pathological characteristics of colorectal polyps in Iranian population. East Afr J Public Health 7: 157-159, 2010.

31. Gschwantler M, Kriwanek S, Langner E, et al: High-grade dysplasia and invasive carcinoma in colorectal adenomas: a multivariate analysis of the impact of adenoma and patient characteristics. Eur J Gastroenterol Hepatol 14: 183-188, 2002.

32. Jacobs ET, Thompson PA and Martínez ME: Diet, gender and colorectal neoplasia. J Clin Gastroenterol 41: 731-746, 2007.

33. Gupta S, Balasubramanian BA, Fu T, Genta RM, Rockey DC and Lash R: Polyps with advanced neoplasia are smaller in the right than in the left colon: implications for colorectal cancer screening. Clin Gastroenterol Hepatol 10: 1395-1401 e1392, 2012 . 